\title{
The Robot: a big disappointment, or just early days?
}

Most articles on robotic colorectal surgery start with describing the limitations of laparoscopic surgery such as suboptimal surgeon ergonomics, assistant dependent 2D camera views and limited dexterity of the instruments. Then the potential advantages of the robot such as excellent ergonomics, tremor elimination, 3D view, motion scaling and instruments with multiple degrees of freedom are mentioned. All these features are said to be ideal for working in a confined space such as the narrow pelvis in an obese male patient, for example.

Numerous retrospective, prospective and case-controlled studies have indeed demonstrated the three steps of a robust evaluation of a new surgical procedure/ technology [1]: feasibility, safety and effectiveness of the robotic system. In addition, these studies resulted in equal or better specimen-oriented outcomes such as quality of TME, CRM, lymph node yield and many more. However, until now, high-quality prospective RCTs have failed to show any benefit. The ROLARR study failed to show any statistical difference in operative, specimen-oriented oncologic or functional outcome including no advantage in regards to conversion [2]. Long-term oncological outcome are still awaited. The Wall Street Journal stated that 'Robot-assisted surgery costs more but may not be better' (Oct. 30, 2017).

Furthermore, there is a more than $80 \%$ 'spin' in publications on robotic colorectal surgery [3]. Spin describes selective reporting that could distort the interpretation of results and mislead readers. The most common forms of spin were 'concluding equivalence based upon non-significant difference $(71 \%$ of abstracts and $76 \%$ of conclusions)' and/or 'claiming improved benefits of the robotic approach despite nonsignificance ( $26 \%$ of abstracts and $45 \%$ of conclusions)'.

The IDEAL framework for new surgical procedures/ devices describes five stages of evaluation: idea, development, exploration, assessment and long-term study [1]. All this has been done for colorectal surgery since the introduction of the robot in 2000 without any clearly proven patient-related benefits. Robot-assisted surgeries are longer and more expensive. Should we now stop using the robot?

Tesla loses money every quarter but keeps on building and testing driverless electric cars. Robotic surgery is constantly evolving along with new robotic technology. New developments will introduce haptic, tactile feedback and smart technology. Artificial intelligence integration into surgical robotics is as inevitable as the autopilot in commercial aviation. Automation will likely be a next step. An orthopedic robotic arm can already today make real-time adjustments in the OR during joint replacements (Scientific American, July 13, 2017). In the future, why not let the robot do the perfect low colo-rectal anastomosis with no leaks?

Right now the robot is not yet autonomous. Thus, surgeons require plenty of training in order to be proficient. We need to focus on effective training curricula. The next two years promise the introduction of new platforms and therefore competition which will hopefully reduce costs. Miniaturization is likely to occur even as the power of the hardware increases. The technology will also incorporate augmented reality to combine the numerous feeds of information currently spread across multiple monitors and systems around patient care. Artificial intelligence will team up with surgical robots. In the future, surgeons will use robotic and smart micro devices on demand to assist and augment procedures. Hypothetically, a sufficiently advanced surgical robot would have access to more data than its human counterpart, and would be able to operate with more precision and a smaller chance or error. Rapid advancements in technology repeatedly remind us to rule-out no possibilities. Until then we need to continue exploring the current available technology which is today 'just' a direct telemanipulator.

Opponents of robotic colorectal surgery stress the fact that just because we can do it with the robot does not mean that we should. Fortunately for the proponents the opposite is also true. If we do not use the robot for colorectal surgery, how are we to know that we should not. The lack of data should not be considered as evidence against the use of this technology, but rather should suggest that further efforts are necessary to establish the role of robotic colorectal surgery. The value of comprehensive data collection through registries at all stages of the IDEAL framework has been recently recognized and is a way to go [1].

We must see the evolution of robotics in colorectal surgery as a journey with meaningful exploration toward greater knowledge and better operations for our patients. The robot is here and will stay. We need to keep on using it.

\section{Dieter Hahnloser}

Department of visceral surgery, University Hospital Lausanne, Lausanne, Switzerland

(a)d_hahnd 


\section{References}

1 Hirst A, Philippou Y, Blazeby J et al. No surgical innovation without evaluation: evolution and further development of the IDEAL framework and recommendations. Ann Surg 2018; https://doi.org/10.1097/SLA.0000000000002794.

2 Jayne D, Pigazzi A, Marshall $\mathrm{H}$ et al. Effect of roboticassisted vs conventional laparoscopic surgery on risk of conversion to open laparotomy among patients undergoing resection for rectal cancer: the ROLARR randomized clinical trial. JAMA 2017; 318: 1569-80.

3 Patel SV, Chadi SA, Choi J, Colquhoun PH. The use of "spin" in laparoscopic lower GI surgical trials with nonsignificant results: an assessment of reporting and interpretation of the primary outcomes. Dis Colon Rectum 2013; 56: 1388-94. 